\title{
Bit and Power Allocation Scheme for Multicarrier Faster-Than-Nyquist Signaling
}

\author{
Hao Duan ${ }^{1}$, Yuanyuan $\mathrm{Gao}^{2}$, Mingxi Guo ${ }^{1}$, Yuehong Shen ${ }^{1}$ \\ ${ }^{1}$ Institute of Communications Engineering, PLA University of Science and Technology \\ ${ }^{2}$ Nanjing University of Posts and Telecommunications \\ 210000, Nanjing, China \\ njduanhao@163.com, yuanyuan.gao@vip.126.com,gogomx@163.com, chunfeng22259@126.com.
}

\begin{abstract}
The transmitter of multicarrier faster-thanNyquist Signaling is a promising direction. In this paper, the bit and power allocation problem is discussed for the multicarrier FTN environment, with the aim to maximum mutual information while guarantee the practicality. An improved spectral shaping scheme with integer bit constraint is proposed for the transmitter. It is based on the modified iterative water-filling and greedy algorithm revised for our scenario. Correspondingly, there are two processes for the scheme called iterative power allocation and discrete bit replenishment. With the benefit of improved method, integer bits can be ensured for each subcarrier at the cost of a little mutual information loss. This is confirmed by simulation and numerical results.
\end{abstract}

Keywords-faster-than-Nyquist; spectral shaping ; integer bit constraint; power allocation; mutual information

\section{INTRODUCTION}

Faster-than-Nyquist (FTN) signaling was first proposed by J. E. Mazo in 1975 [1]. It has recently gained new attention due to the advantage of high information rate and better bandwidth efficiency, which caters to people's requirement in our bandwidth-starved world. Different from traditional intersymbol interference (ISI) free transmission model, symbols are sent faster than the Nyquist rate in the scenario of FTN signaling. So the pulses are non-orthogonal and it has pushed the limit of the Nyquist ISI free criterion. Later this was extended to the multicarrier system like OFDM, in which the interference can be introduced to both time and frequency domain [2].

Compared to the study of FTN receivers, research on whose transmitting terminal is little. Mazo chooses the sinc pulse initially and proved that the rate could exceed $25 \%$ of the Nyquist rate without reducing the minimum Euclidean distance for the binary case. Considering the ideal low-pass filter is difficult to be physically realized, some other forms of pulse are exploited in recent years, such as the raised cosine and root raised cosine pulses [3][4][5]. In this process, a higher Shanon capacity of FTN signaling gradually attracts people's interest. In [4], Rusek and Anderson explored all kinds of signals such as the family of root raised cosine pulses. They show that the capacity of FTN is higher than that of signals based on orthogonal linear modulation for almost all pulses except the sinc.

This work is supported by the National Natural Science Foundation of China (No.61301157).

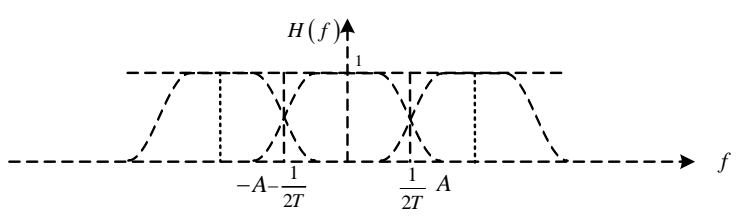

Fig. 1. Transmission of signals at Nyquist rate.

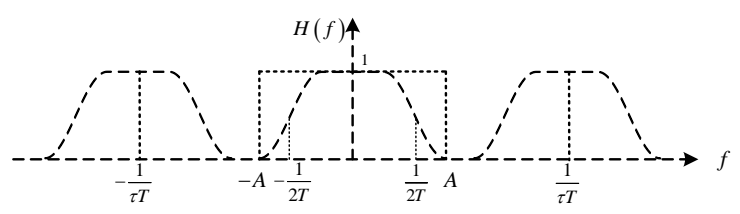

Fig. 2. Transmission of signals at FTN rate.

Figure 1 and Figure 2 shows the transmission of signals at Nyquist rate $1 / T$ and at FTN rate $1 / \tau T$ respectively, where $\tau$ is the acceleration factor and satisfies $0<\tau<1$ [5]. The constrained capacity of FTN signaling is [4]

$$
C_{F T N}=\int_{0}^{A} \log _{2}\left(1+\frac{2 P}{N_{0}}|H(f)|^{2}\right) d f
$$

where $A$ is the bandwidth of signal, $P$ is the average transmit power, $N_{0}$ is the noise power spectral density, $H(f)$ is the spectrum of pulse $h(t)$ having unit energy.

Channel capacity is the ideal highest information rate for reliable communication. Some recent researches concentrate on applying spectral shaping to the FTN scenario, so as to improve the mutual information. In [6], water-filling algorithm is first studied in binary FTN signaling to achieve the channel capacity asymptotically . With receiver mismatch, Y. Kim and J. Bajcsy have discussed the maximal information rate of cyclostationary FTN system [7].

A noteworthy study is the spectral shaping for multicarrier FTN signaling in [8]. Hefnawy and Dietl investigate how to optimize the mutual information of FTN transmit model. They employ both input and output constraints to the transmit filter to guarantee the practicality. By use of Lagrangian multiplier method, they get the optimal power allocation scheme. However, just as the classical water-filling, the spectral shaping method in this paper ignores the subcarriers' practical modulate constellation. As a result, the obtained number of bits allocated to each subcarrier may be decimals, which violates the 
practical situation. In view of this problem, we propose an improved scheme with the integer bit constraint to all subcarriers. Our contributions are summarized as follows:

- We discuss the bit and power allocation problem in the multicarrier FTN environment and formulate the target function to maximum the information rate while guarantee the practicality.

- An improved spectral shaping scheme is proposed based on the modified iterative water-filling and greedy algorithm suitable for multicarrier FTN scenario, which consists of two processes called iterative power allocation and discrete bit replenishment.

- Numerical results are provided to demonstrate the advantage and effectiveness of the proposed scheme.

\section{SYSTEM DESCRIPTION}

Block diagram of the considered multicarrier FTN system is shown in Figure 3 just as in [8], where the channel is interpreted as a RRC transmit filter using FTN signaling. The available channel bandwidth $A$ is divided into $K$ subcarriers with the relatively narrow width $\Delta f=A / K$.

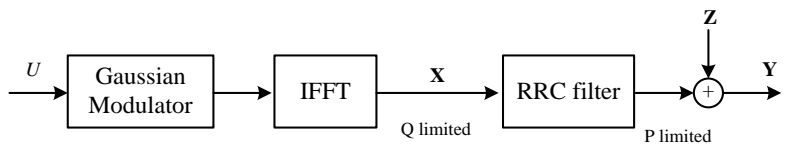

Fig.3. System model of FTN transmission

So the $k$ th subcarrier $f_{k}$ equals to $(2 k-K-1) \Delta f / 2$. $U$ is the vector of random bits that pass through a Gaussian modulator which can insure the zero-mean independent Gaussian inputs of RRC filter. It then generates $K$ symbols to the common inverse fast Fourier transform (IFFT) block. After being shifted to the subcarriers, symbols constitute the input vector $\mathbf{X}$ of the transmit filter, where $\mathbf{X}=\left(X_{1}, X_{2}, \ldots, X_{K}\right)$. With the Gaussian noise $\mathbf{Z}$, the output vector of the system is $\mathbf{Y}=\left(Y_{1}, Y_{2}, \ldots, Y_{K}\right)$. We have

$$
Y_{k}=H\left(f_{k}\right) X_{k}+Z_{k}
$$

where $H(f)$ is the channel transfer function of RRC pulse whose equivalent bandwidth is $W=A /(1+\alpha)$. The $Z_{k}$ are zero-mean independent Gaussian noise symbols. The power spectral density of Gaussian input $X_{k}$ is $Q_{k}$. According to this transmit model, the upper bound of mutual information can be computed as

$$
I(\mathbf{X}, \mathbf{Y}) \leq \sum_{k=1}^{K} \log _{2}\left(1+\frac{\left|H\left(f_{k}\right)\right|^{2} Q_{k}}{N_{0}}\right)=C(\mathbf{Q})
$$

where $\mathbf{Q}=\left(Q_{1}, Q_{2}, \ldots, Q_{k}\right)$.

To maximum (3), two power constraints are taken into consideration as below

$$
\sum_{k=1}^{K} Q_{k}\left|H\left(f_{k}\right)\right|^{2} \Delta f \leq P
$$

$$
\sum_{k=1}^{K} Q_{k} \Delta f \leq Q
$$

where $P$ is the transmit power constraint after the pulse shaper, $Q$ is the input power constraint. If considering only this constraint, the best spectral shaping we can get is an inverse filter by means of Lagrangian multiplier method. But this may require a large power at the input of the transmit filter, so the constraint (5) seems indispensable. The solution is given as

$$
Q_{k}=\max \left(0, \frac{\log _{2} e}{\delta+\gamma\left|H\left(f_{k}\right)\right|^{2}}-\frac{N_{0}}{\left|H\left(f_{k}\right)\right|^{2}}\right)
$$

where $\delta$ and $\gamma$ are Lagrangian multipliers depending on $Q$ and $P$ respectively.

This solution of spectral shaping seems to be the optimum power distribution to maximum the mutual information definitely. Nevertheless, we should observe that the scheme is just a theoretical optimal result like the classical water-filling algorithm, without considering the size of the modulate constellation. So the number of bits transmitted by each subcarrier may not be integer. This is unpractical obviously. In view of this problem, we put forward an improved scheme which is demonstrated in the following section.

\section{PROBLEM FORMULATION AND IMPROVED SCHEME}

According to [8], we can observe that the upper bound of information rate for the $k$ th subcarrier is

$$
b_{k}=\log _{2}\left(1+\frac{\left|H\left(f_{k}\right)\right|^{2} Q_{k}}{N_{0}}\right)
$$

However, the value of $b_{k}$ is not necessarily integers as mentioned before. Hence we have to round it down to the nearest integer as below [9].

$$
\hat{b}_{k}=\left\lfloor b_{k}\right\rfloor
$$

where $\hat{b}_{k}$ is the actual number of bits can be carried by subcarriers, which corresponds to the required power density as

$$
\hat{p}_{k}\left(b_{k}\right)=\left(2^{b_{k}}-1\right) N_{0} /\left|H\left(f_{k}\right)\right|^{2}
$$

So the whole allocation problem with integer bit constraint for multicarrier FTN system can be formulated as follows

$$
\left.\begin{array}{ll} 
& \max (B) \\
\text { s.t. } & \sum_{k=1}^{K} \hat{p}_{k}\left(\hat{b}_{k}\right) \leq Q \\
& \sum_{k=1}^{K} \hat{p}_{k}\left(\hat{b}_{k}\right) \cdot\left|H\left(f_{k}\right)\right|^{2} \leq P
\end{array}\right\}
$$

where $B=\sum_{k=1}^{K} \hat{b}_{k}$ is the whole practical mutual information.

In order to solve this new problem, we propose an improved spectral shaping scheme based on the modified iterative water-filling and greedy algorithm for the scenario of multicarrier FTN signaling . There are two stages during the procedure which can be called iterative power allocation and 
discrete bit replenishment. The specific depiction of the scheme is shown as follows:

Stage 1 : iterative power allocation

Learning from the thought in iterative water-filling algorithm, we modify it to be suitable for our multicarrier FTN environment, which consists of four steps as follows.

Step (1): Take the initial power allocation based on the conclusion given in [8]. The input power $Q$ should be allocated to all the subcarriers firstly. According to the Lagrangian multiplier method, the power spectral density for the $k$ th subcarrier should be

$$
Q_{k}=\log _{2} e /\left(\delta+\gamma\left|H\left(f_{k}\right)\right|^{2}\right)-N_{0} /\left|H\left(f_{k}\right)\right|^{2}
$$

Step (2): Reallocate the power to the subchannels iteratively. Firstly, search for the subcarriers whose allocation values are negative and then adjust them to zero. Meanwhile, include these subcarriers to the set $\bar{U}$ which covers all the unusable subcarriers. Then the set of usable subcarriers $U$ can be obtained, within the scope of which we can recompute the allocation scheme $Q_{k}(k \in \mathrm{U})$ until $Q_{k}>0(\forall k \in \mathrm{U})$.

Step (3): Compute the upper bound of information bits for each subcarriers and then round them down to the nearest integer. The calculation formulas are (7) and (8).

Step(4): Compute the required total power $\hat{Q}$ until step 3. According to (9) and (10), we can derive that the remaining input power $Q_{e}$ and the corresponding transmit power $\hat{P}$ as follows.

$$
\begin{gathered}
\hat{Q}=\sum_{k=1}^{K} \frac{N_{0}}{\left|H\left(f_{k}\right)\right|^{2}}\left(2^{\hat{b}_{k}}-1\right) \\
\hat{P}=\sum_{k=1}^{K} \frac{N_{0}}{\left|H\left(f_{k}\right)\right|^{2}}\left(2^{\hat{b}_{k}}-1\right)\left|H\left(f_{k}\right)\right|^{2} \\
=\sum_{k=1}^{K} N_{0}\left(2^{\hat{b}_{k}}-1\right) \\
Q_{e}=Q-\hat{Q}
\end{gathered}
$$

Stage 2: discrete bit replenishment

Allocate the remaining power to each subcarrier by the improved greedy algorithm on the basis of multicarrier FTN environment, which consists of two stages as follows.

Step (1): Compute the additional power while adding just one bit to each subcarrier in set $U$.

$$
\begin{gathered}
\Delta Q_{k}=\frac{N_{0}}{\left|H\left(f_{k}\right)\right|^{2}} 2^{b_{k}} \\
\Delta P_{k}=\frac{N_{0}}{\left|H\left(f_{k}\right)\right|^{2}} 2^{b_{k}}\left|H\left(f_{k}\right)\right|^{2}=2^{b_{k}} N_{0}
\end{gathered}
$$

Step (2): Search for the suitable $k^{*}=\underset{1 \leq k \leq K}{\arg \min } \Delta Q_{k}$, judging whether meet the inequalities $\hat{Q}+\Delta Q_{k^{*}} \leq Q$ and $\hat{P}+\Delta P_{k} \leq P$. If they holds, back to step (1). Otherwise, the loop stops.

\section{PERFORMANCE EVALUATION}

In this section, some numerical results will be given to demonstrate the effectiveness and advantage of the proposed improved method. We assume an multicarrier FTN system with parameters $K=1024, T=1 / 2, A=3, \alpha=0.5$ and $\tau \leq 1 / A T=2 / 3$ to avoid aliasing. One of the constraints $Q$ for spectral shaping is limited to $10^{3}$.

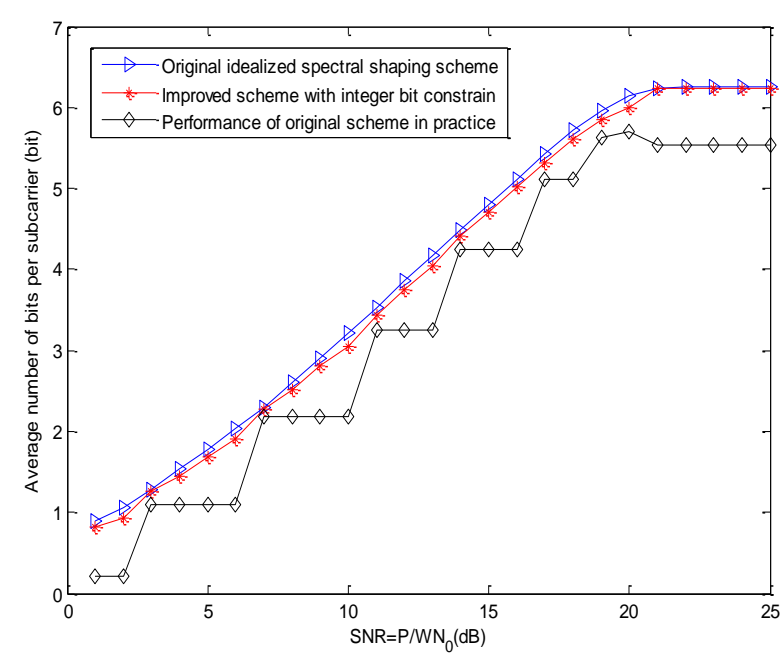

Fig.4 Performance comparision of original and improved scheme

Figure 4 compares the average number of bits for each subcarrier of original and our proposed method. We can conclude that the information rate of improved scheme is slightly less than the original one, for it is an ideal case without considering integer bit constraint. The practical performance of the original scheme without improvement is also given, which is obviously inferior.

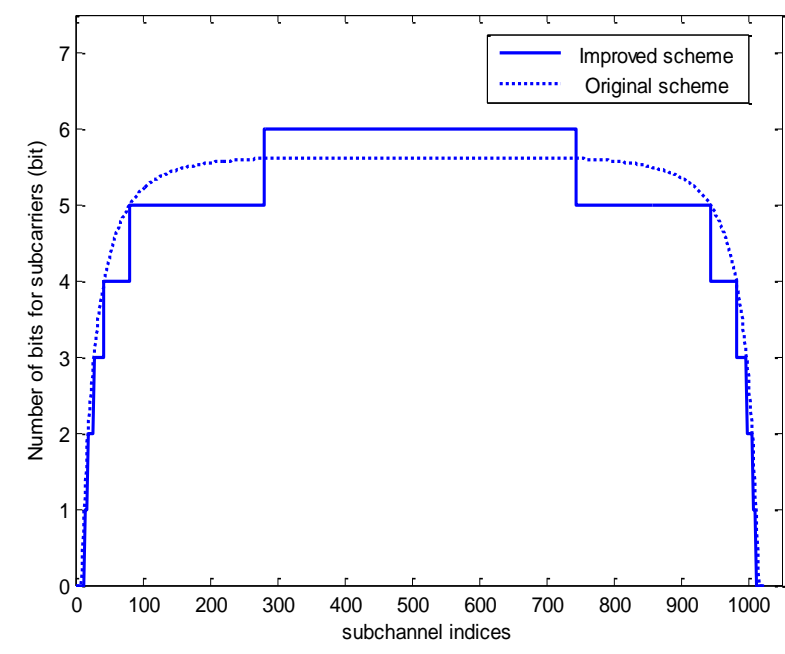

Fig. 5 Number of bits transmitted by each subcarrier

Figure 5 shows the number of bits transmitted by each subcarrier when $\mathrm{SNR}=18 \mathrm{~dB}$. It can be observed that the improved scheme we put forward satisfies the integer bit constraint. 


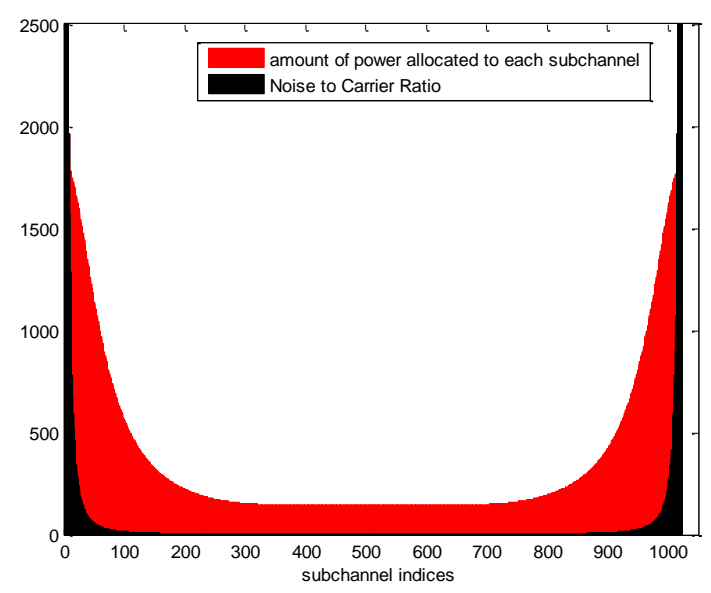

Fig.6 Power allocation for original scheme

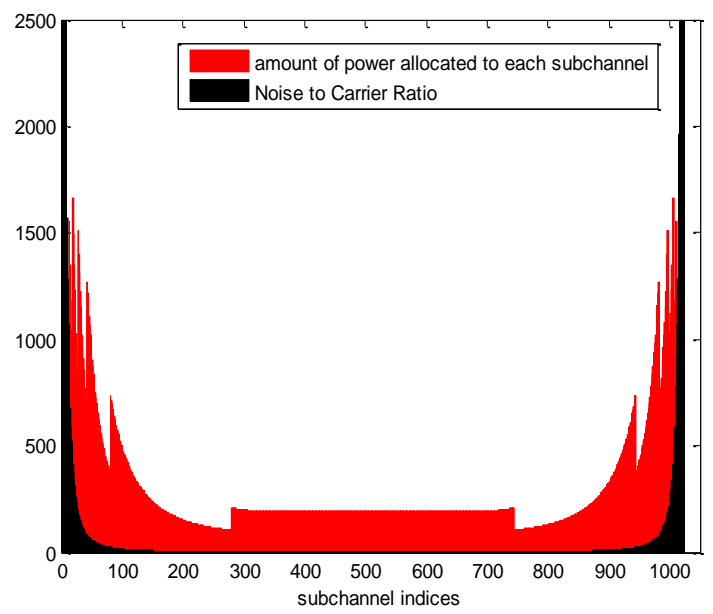

Fig.7 Power allocation for improved scheme

The simulation results of the power allocation when $\mathrm{SNR}=18 \mathrm{~dB}$ are shown in figure 6 and Figure 7. Actually, with the increase of constraint $P$ in Figure 6, the amount of power allocated to each subchannel will gradually distribute as a horizontal line like the water pouring to the bowl, because the scheme gradually transforms into the classical waterfilling criterion during this process. It is different while using our integer bit constraint method, which is shown in Figure 7. It can be seen that the power distribution of these subchannels presents a zigzag appearance, for we improve the original scheme to ensure integer bit upper bound of each subcarrier. In addition, we can observe that the total amount of power allocated to subcarriers in the improved scheme, which is actually the red area in Figure 7, is slightly less than that in original scheme, because we have to sacrifice a little power to ensure the overall performance eventually.

\section{CONCLUSION}

In this paper, we discuss the bit and power allocation problem in the multicarrier FTN environment. With the given transmit model in [8], we formulate the objective function to maximum the information rate while guarantee the practicality. Afterwards an improved spectral shaping scheme with integer bit constraint is proposed for the transmitter. This improved method is based on the modified iterative water-filling and greedy algorithm adapted to our scenario. According to the numerical results, we can conclude that the proposed improved method is superior to the original one, for the integer bits can be ensured with just a little cost.

\section{REFERENCES}

[1] J. E. Mazo, “Faster-than-Nyquist signaling,'” Bell Syst. Tech. J., vol. 54, pp. 1451-1462, Oct. 1975.

[2] F. Rusek and J. B. Anderson, "The two dimensional Mazo limit," in Proc. IEEE Int. Symp. Inf. Theory, Adelaide, Australia, pp. 970-974, Sep. 2005

[3] A. D. Liveris and C. N. Georghiades, "Exploiting faster-than-Nyquist signaling," IEEE Trans. Commun., vol. 51, no. 9, pp. 1502-1511, Sep. 2003.

[4] F. Rusek and J. B. Anderson, "Constrained capacities for faster than Nyquist signaling," IEEE Trans. Inf. Theory, vol. 55, no. 2, pp. 764-775, Feb. 2009.

[5] J. Anderson, F. Rusek, and V. O" wall, "Faster-than-Nyquist signaling," Proc. IEEE, vol. 101, no. 8, pp. 1817-1830, Aug. 2013.

[6] Y. G. Yoo and J. H. Cho, “Asymptotic optimality of binary faster-thanNyquist signaling,' IEEE Commun. Lett., vol. 14, no. 9, pp. 788-790, Sep. 2010.

[7] Y. Kim and J. Bajcsy, "Information rates of cyclostationary Faster-thanNyquist signaling", 12th Can. Workshop Inf. Theory (CWIT). IEEE, pp. 1-4, May. 2011.

[8] M. E. Hefnawy and G.Dietl, "Spectral Shaping for Faster-than-Nyquist Signaling", Wireless Communications Systems (ISWCS), International Symposium on. IEEE, pp. 496 - 500, Aug. 2014.

[9] J. Y. Xue and B. L. Jiao. "An improved bit and power allocation algorithm for OFDM systems". Acta Scientiarum Naturalium Universitatis Pekinensis, vol.42, no.1, pp. 93-98, Feb. 2006. 